\title{
ON THE FORMATION OF THE ASTEROIDS
}

\author{
J. G. $H I L L S$ \\ University of Michigan
}

Recently Anders (1965) has shown that the smaller asteroids are overabundant relative to their initial numbers because of the collisional fragmentation of larger asteroids. Allowing for this fragmentation, he has derived a reconstructed initial radius distribution. Although the reconstructed distribution only comprises a little more than 100 asteroids and consequently has a large statistical uncertainty as well as the uncertainty introduced by the reconstruction process, Anders finds that when displayed on a log-log plot, the distribution can be represented fairly well by a gaussian curve with a peak near radius $R=30 \mathrm{~km}$. This work has basically been substantiated by Hartmann and Hartmann (1968), although Hartmann (1968) notes that a gaussian distribution underestimates the observed number of more massive asteroids. This suggests that the initial asteroid distribution function was probably broader than a gaussian function, but it still retains a distinct bell-like appearance.

In this paper we propose a simple model for the accretion of objects in the solar nebula that permits a straightforward calculation of their radius function. This fits the Anders reconstructed asteroid distribution, and it also predicts reasonably well the number of terrestrial planets. (The terrestrial planets are assumed to be asteroids that formed slightly earlier than their fellows and consequently captured most of the available solid material in the solar nebula. Subsequently the asteroids and terrestrial planets will collectively be called planetoids.) A number of consequences of our model are explored in this paper. A preliminary report on this theory has been published (Hills, 1970).

\section{RADIUS FUNCTION}

The calculation of the radius function of the planetoids requires some knowledge of the rate of formation of the seed bodies that initiated their accretion. From knowledge of other processes requiring the formation of seed bodies, it is probable that their formation was governed by a stochastic process. In this case, the rate of formation of the seed bodies was independent of time as long as the total mass accumulated in the planetoids remained much smaller than the amount of unaccreted material.

With the rate of formation of the seed bodies being independent of time, the number of planetoids with radii between $R$ and $R+\Delta R$ is directly 
proportional to the time necessary for the radius of a planetoid to grow from $R$ to $R+\Delta R$. This requires a radius distribution function of the form

$$
d N(R)=\frac{N_{0}^{\prime} d R}{d R / d t}
$$

where the constant of proportionality $N_{0}{ }^{\prime}$ is the number of seed bodies formed per unit time in the nebula, and $d R / d t$ is determined by the accretion equation. (See, e.g., Hartmann, 1968.)

$$
\frac{d R}{d t}=\alpha \frac{\rho_{a}}{\rho_{p}} \frac{V}{4}\left(1+\frac{8 \pi G R^{2} \rho_{p}}{3 V^{2}}\right)
$$

Here $\alpha$ is the sticking coefficient, $\rho_{a}$ is the space density of the accretable material, $\rho_{p}$ is the planetoid density, and $V$ is the average preencounter velocity of the accreted particles relative to the planetoid. The equation is simplified by introducing a characteristic radius,

so that

$$
R_{c}=\left(\frac{3}{8 \pi G \rho_{p}}\right)^{1 / 2} V
$$

$$
\frac{d R}{d t}=\alpha \frac{\rho_{a}}{\rho_{p}} \frac{V}{4}\left[1+\left(\frac{R}{R_{c}}\right)^{2}\right]
$$

$R_{c}$ is the radius at which the accretion cross section of a planetoid is twice its geometric cross section.

Making use of the accretion equation, the radius distribution function becomes

$$
d N(R)=\frac{N_{0} d R / R_{c}}{1+\left(R / R_{c}\right)^{2}}
$$

where the new constant of proportionality is

$$
N_{0}=\frac{4 N_{0}^{\prime} R_{c}}{\alpha V} \frac{\rho_{p}}{\rho_{a}}
$$

Integrating equation (5), we find that the number of planetoids with radii equal to or less than $R$ is

As $R \rightarrow \infty$,

$$
N(R)=N_{0} \tan ^{-1} \frac{R}{R_{c}}
$$

$$
N(\infty)=N_{0} \frac{\pi}{2}
$$

Thus the number of planetoids is formally bound even if the radius of the largest one and the total mass of the system are not. This results from the 
accretion cross section of the largest object formally growing much faster than its mass, which allows it to grow to infinite mass in a finite time if enough material is present. In a real system, the number of planetoids is similarly not determined by the total mass of the system but by the ratio of the time necessary for the largest object in the system to acquire most of its mass to the average time between the production of the seed bodies. Most of the mass in a typical planetoid system will be accumulated in the first one or two largest bodies.

In any actual system there is an upper limit $R_{\max }$ to the radius of the largest planetoid, but if $R_{\mathrm{max}} \gg R_{c}$, then $N(\infty) \sim N\left(R_{\mathrm{max}}\right)$. If we mathematically allow $R \rightarrow \infty$ so that $N(\infty)$ is the total number of planetoids formed, the normalized integrated radius function is

$$
N(R)=\frac{2}{\pi} N(\infty) \tan ^{-1} \frac{R}{R_{c}}
$$

and in differential form

$$
d N(R)=\frac{2}{\pi} N(\infty) \frac{d R / R_{c}}{1+\left(R / R_{c}\right)^{2}}
$$

We note that $R_{c}$ is the median radius of the planetoids.

To compare the theoretical radius distribution function with the Anders distribution we have to convert the former into one in units of $\ln R$. This yields

$$
d N(\ln R)=\frac{2}{\pi} N(\infty) \frac{\left(R / R_{c}\right) d\left[\ln \left(R / R_{c}\right)\right]}{1+\left(R / R_{c}\right)^{2}}
$$

This function is plotted in figure 1. It is a serpentine curve and looks quasi-gaussian about the peak at $R=R_{c}$.

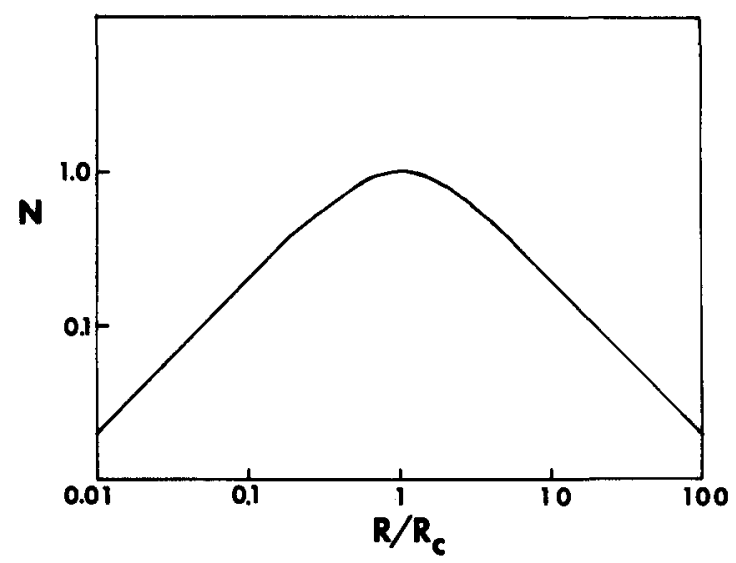

Figure 1.-Theoretical radius function of the planetoids. 
The theoretical curve fits the reconstructed radius distribution to within the statistical errors if $R_{c} \sim 15 \mathrm{~km}$. This function is noticeably broader than a gaussian function. For the initial asteroid system, the radius of Ceres is $R_{\max }$. We note in passing, that if we normalized the theoretical radius function to the reconstructed asteroid radius distribution, any planetoids with $R>R_{\max }$ predicted by the theoretical relation can only have a mathematical and no physical significance.

With $R_{c}=15 \mathrm{~km}$ and $\rho_{p}=3.6 \mathrm{~g} / \mathrm{cm}^{3}$ for the asteroids we find by equation (3) that $V=0.02 \mathrm{~km} / \mathrm{s}$. The peak of Anders' (1965) proposed empirical function is $R_{c} \sim 30 \mathrm{~km}$ with an error of about 50 percent. For $R_{c}=30 \mathrm{~km}, V=0.04 \mathrm{~km} / \mathrm{s}$. We take $V=2 \times 10^{-2}$ to $4 \times 10^{-2} \mathrm{~km} / \mathrm{s}$ as the likely range of $V$. This $V$ was presumably due to large-scale turbulent motion in the solar nebula.

We shall now investigate some consequences of our theoretical model to shed more light on the planetoid formation process and to better test the validity of the theory by producing a larger body of results to check against observable data.

\section{TOTAL NUMBER OF PLANETOIDS}

Assuming that $V$ was constant throughout the solar nebula allows the calculation of the total number $N(\infty)$ of planetoids formed in the region of the solar nebula now occupied by the terrestrial planets (terrestrial band). The total mass of a system of planetoids in which the largest body has a mass $M_{\max }$ is found by integrating equation (10). This gives

$$
M_{\text {total }}=\int_{0}^{R_{\max }} \frac{2}{\pi} N(\infty) \frac{4}{3} \pi R^{3} \rho_{p} \frac{d R / R_{c}}{1+\left(R / R_{c}\right)^{2}}
$$

On completing the integration and rearranging terms, we find

$$
N(\infty)=\frac{\pi\left(M_{\text {total }} / M_{c}\right)}{\left(M_{\max } / M_{c}\right)^{2 / 3}-\ln \left[1+\left(M_{\max } / M_{c}\right)^{2 / 3}\right]}
$$

where from equation (3)

$$
M_{c}=\frac{4}{3} \pi R_{c}^{3} \rho_{p}=\left(\frac{3}{32 \pi G^{3} \rho_{p}}\right)^{1 / 2} V^{3}
$$

Because $M_{\max } \gg M_{c}$,

$$
N(\infty) \sim \pi \frac{M_{\text {total }}}{M_{\max }{ }^{2 / 3} M_{c}^{1 / 3}}
$$

We note that $N(\infty) \alpha \rho_{p}{ }^{1 / 6}$. This weak dependence of $N(\infty)$ on $\rho_{p}$ makes rather immaterial whether we use $\rho_{p}=3.6 \mathrm{~g} / \mathrm{cm}^{3}$ as for chondrite meteorites or 
$\rho_{p}=5.5 \mathrm{~g} / \mathrm{cm}^{3}$ as for Earth or Mercury in calculating $N(\infty)$. For the terrestrial band, $M_{\text {total }}=2.0 M_{\oplus}$ (total mass of present terrestrial planets and the asteroids) and $M_{\max }=1.0 M_{\oplus}$. With these values for the masses and $V=0.02 \mathrm{~km} / \mathrm{s}, N(\infty)=3508$ for $\rho_{p}=5.5 \mathrm{~g} / \mathrm{cm}^{3}$ and 3269 for $\rho_{p}=3.6 \mathrm{~g} / \mathrm{cm}^{3}$; whereas for $V=0.04 \mathrm{~km} / \mathrm{s}, N(\infty)=1752$ for $\rho_{p}=5.5 \mathrm{~g} / \mathrm{cm}^{3}$ and 1633 for $\rho_{p}=3.6 \mathrm{~g} / \mathrm{cm}^{3}$.

\section{ACCRETED PLANETOIDS}

Table I shows the normalized planetoid mass distribution functions for $\rho_{p}=3.6 \mathrm{~g} / \mathrm{cm}^{3}$ and turbulent velocities of 0.02 and $0.04 \mathrm{~km} / \mathrm{s}$. Note that the mass distributions are almost the same for masses greater than $10^{-5} M_{\oplus}$. One can show that the number of these more massive objects is similarly insensitive to $\rho_{p}$. Presumably, except for about 100 initial asteroids and their fragments, the smaller planetoids have been accreted by the four remaining terrestrial planets and the Moon.

From the table we find that about 15 percent of the mass of the original planetoid system was in objects less massive than Mercury, which implies that about 15 percent of the mass of Earth and the other terrestrial planets was accreted as small planetoids, whereas the remaining 85 percent was accreted as subplanetoid bodies, primarily clumps of dust. About one-third the mass of the accreted planetoids was in objects having sublunar masses and two-thirds was in objects having masses between that of the Moon and Mercury.

We may be concerned that a collision between a large planetoid in the latter group and a terrestrial planet could cause their mutual destruction. A breakup is expected if the preencounter total kinetic energy of the two objects relative to their center of mass is greater than their combined gravitational binding energies; i.e., for planetoids of uniform density, breakup requires that

$$
\frac{1}{2} \mu V^{2}>\frac{3}{5} G\left(\frac{M_{1}{ }^{2}}{R_{1}}+\frac{M_{2}^{2}}{R_{2}}\right)
$$

where $\mu=\left(M_{1} M_{2}\right) /\left(M_{1}+M_{2}\right)$, the reduced mass, and $V$ is the relative velocity of the two objects prior to the encounter. An upper limit on $V$ is probably its present value for interasteroidal collisions, $5 \mathrm{~km} / \mathrm{s}$ (Piotrowski, 1953). After the terrestrial planets formed, their long-range gravitational perturbations increased $V$ well above the $0.04 \mathrm{~km} / \mathrm{s}$ maximum turbulent velocity in the solar nebula, but these forces were not likely to have had sufficient time before the accretion of most of the small planetoids to raise $V$ much above its present interasteroidal value. Figure 2 shows the mass $m$ of the smallest body required to cause the collisional breakup of a planetoid of mass $M$ if $V=5 \mathrm{~km} / \mathrm{s}$ and $\rho_{p}=5.5 \mathrm{~g} / \mathrm{cm}^{3}$ or $3.6 \mathrm{~g} / \mathrm{cm}^{3}$. We note that planetoids more massive than $0.026 M_{\oplus}$ for $\rho_{p}=5.5 \mathrm{~g} / \mathrm{cm}^{3}$ and $0.033 M_{\oplus}$ for $\rho_{p}=3.6 \mathrm{~g} / \mathrm{cm}^{3}$ are safe against breakup in collisions with all objects equal to or less massive than themselves. As the critical mass is less than half that of Mercury (for $\rho_{p} \sim 5.5$ ), it seems likely that all terrestrial planets were safe against collisional breakup. 


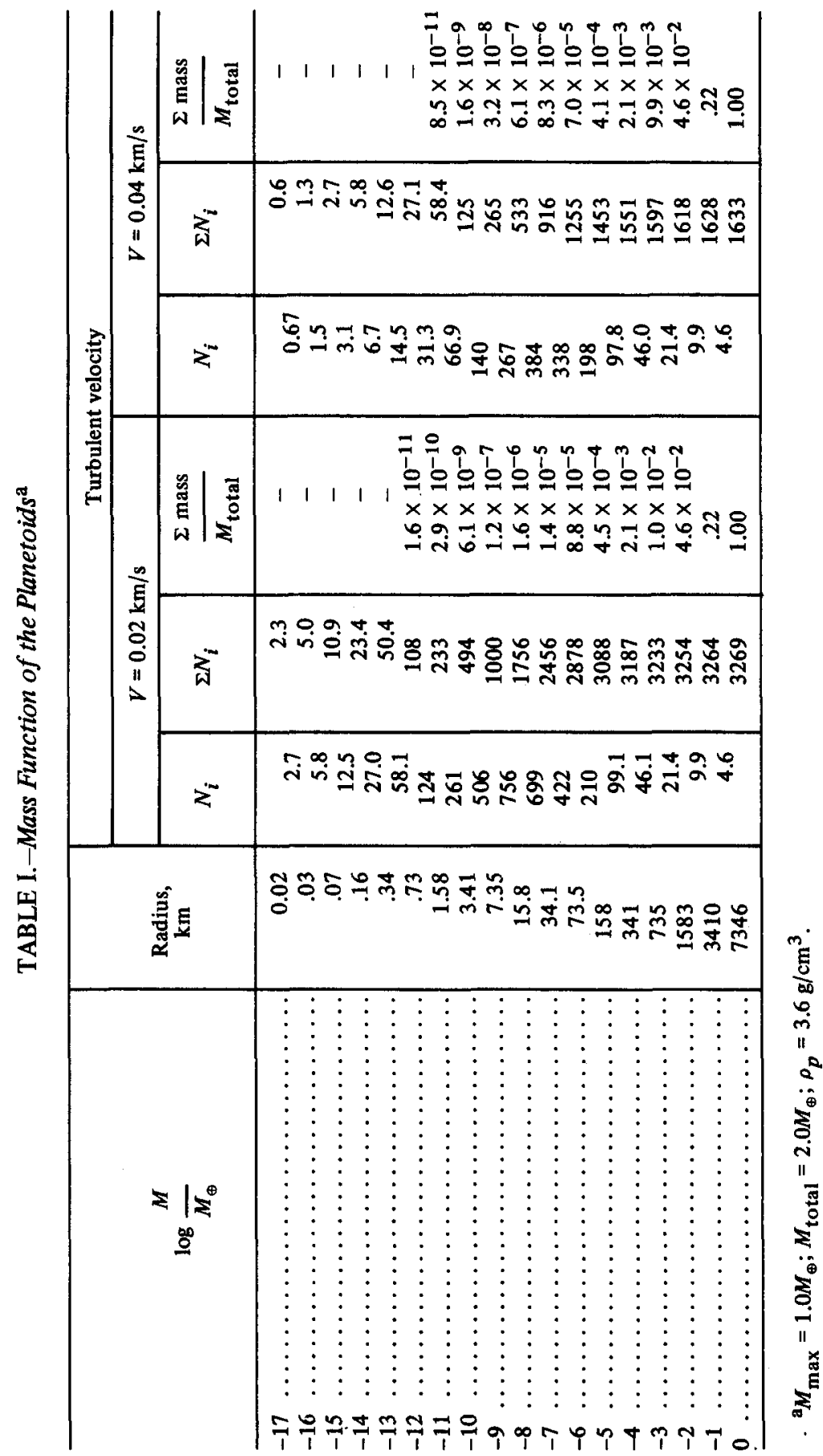




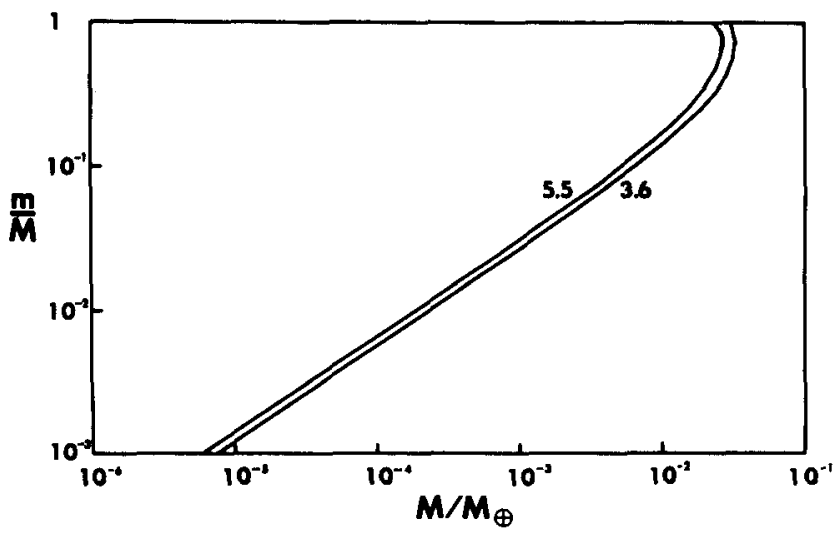

Figure 2.-In a collision between two planetoids having a preencounter relative velocity of $5 \mathrm{~km} / \mathrm{s}, m$ is the minimum mass of the collision partner required to cause the breakup of a planetoid of mass $M$. We note that near the limit of $M$ beyond which a planetoid is stable against breakup irrespective of $m, m$ is double valued, which indicates that there is an upper as well as a lower limit on the $m$ required for fragmentation. This results from the quadratic dependence of the gravitational potential energy on $m$ and $M$.

Although a collision with a large fellow planetoid would not destroy a terrestrial planet, it would produce a drastic alteration in the direction of its rotational axis if the orbit of the planetoid did not lie in the equatorial plane of the planet. ${ }^{1}$ A simple calculation shows that even single collisions with planetoids of lunar mass would easily account for the magnitude of the deviation of the equators of the terrestrial planets from the planes of their orbits. From the theoretical radius distribution function (table I) we see that Earth is likely to have accreted several such bodies.

\section{COLLISIONAL FRAGMENTATION OF THE PLANETOIDS}

Their small number $\left[N(\infty) \sim 2 \times 10^{3}\right.$ to $\left.3 \times 10^{3}\right]$ indicates that the accretion of primary planetoids only produced a very small fraction of the observed lunar and Martian craters. As we shall see, likely agents for the production of most craters are the collisional fragments of a few primary planetoids of approximately lunar mass. We note from figure 2 that lunar-sized objects can be broken apart by collisions with bodies an order of magnitude less massive than themselves, whereas objects the size of Ceres can be broken up in collisions with bodies less than two orders of magnitude less massive than themselves. This extreme fragility of the asteroids suggests an explanation for their failure to coalesce into one body. If an object of $0.03 M_{\oplus}$ or greater had formed in the asteroid belt, we would likely see only one object today.

\footnotetext{
${ }^{1}$ See p. 259.
} 
The average number of fragmentation collisions that occur among a group of $n$ planetoids before they are accreted by the planets is

where

$$
N=\frac{K}{\sigma_{p}}
$$

$$
K=\sum_{i=1}^{n-1} \sum_{i=j+1}^{n} \sigma_{i j}=\frac{1}{2} n(n-1)<\sigma_{i j}>
$$

Here $\sigma_{p}$ is the total : cretion cross section of the terrestrial planets for the average planetoid and $\sigma_{i j}$ is the collision cross section for encounters between planetoids $i$ and $j$. With $V=5 \mathrm{~km} / \mathrm{s}$, the collisional cross sections of sublunar planetoids are very nearly their physical cross sections. Thus

$$
\sigma_{i j} \simeq \pi\left(R_{i}+R_{j}\right)^{2}
$$

Because the radius of the average sublunar planetoid is small compared with the radius $S_{i}$ of a terrestrial planet, by conservation of energy and momentum we find

$$
\sigma_{p} \simeq \sum_{i=1}^{4} S_{i}^{2}\left[\left(\frac{w_{i}}{V}\right)^{2}+1\right]
$$

where $W_{i}$ is the escape velocity from the $i$ th planet. For $V=5 \mathrm{~km} / \mathrm{s}$, $\sigma_{p}=1.5 \times 10^{9} \mathrm{~km}^{2}$.

Table $\mathrm{I}$ indicates that there were 21.4 original planetoids in the mass range $0.001 M_{\oplus}$ to $0.01 M_{\oplus}$. These have radii between 735 and $1580 \mathrm{~km}$ for $\rho_{p}=3.6 \mathrm{~g} / \mathrm{cm}^{3}$. For these objects

$$
<\sigma_{i j}>\simeq \pi(735+1580)^{2}=1.7 \times 10^{7} \mathrm{~km}
$$

From equations (17) through (20) we find that $N=2.4$. Thus it is highly likely that at least one fragmentation collision took place among the objects in this mass range with each collision causing the breakup of two objects.

The observed fragments produced by asteroidal collisions have an integrated radius function of approximately the form (Hartmann and Hartmann, 1968)

$$
N(R)=\left(\frac{R_{\mathrm{max}}}{R}\right)^{2}
$$

where $N(R)$ is the number of fragments with radii larger than $R$. The largest fragment, of radius $R_{\max }$, has usually about one-half the initial mass of the fragmented planetoid. For the objects we have considered, $R_{\max }$ is typically $1000 \mathrm{~km}$ which implies the production of about $10^{6}$ fragments with radii 
greater than $1 \mathrm{~km}$. This is three orders of magnitude larger than the number of primary planetoids, and it is quite adequate to account for the number of large lunar craters. Because $N(R)$ is very sensitive to $R_{\max }$, we can expect that only the first one or two largest fragmented planetoids produced a majority of all the fragments. This result suggests that although most of the integrated mass in planetoids and their fragments accreted by a terrestrial planet or the Moon was in the form of a handful of very large unfragmented primary objects, the vast majority of crater-forming bodies were fragments of a few primary planetoids with initial masses on the order of that of the Moon. If meteorites are fragments of planetoids that were formed in the vicinity of Earth rather than objects that have diffused in from the asteroid belt, we can expect most of them to be from a few primary objects with masses on the order of that of the Moon.

Because the integrated cross-sectional area of the fragments of a planetoid is much larger than its initial cross-sectional area, one collisional fragmentation produces a chain reaction of further fragmentations. Thus, although the integrated mass in planetoids and their fragments that are being accreted by a planet per unit time decreases exponentially with time, an accelerating pace of further fragmentation actually causes the number of objects being accreted per unit time to increase. This is accompanied by a rapid decrease in the average mass of the individual fragments.

The integrated accretion cross section $\sigma_{p}$ of the Jovian planets is about three orders of magnitude greater than that of the terrestrial planets. Consequently, if we apply the same arguments to the planetoids that formed in the region of the Jovian planets that we did to those in the terrestrial band, we find that no fragmentation collisions are likely to have occurred among these planetoids before they were accreted by the Jovian planets. Thus the surfaces of the satellites of the Jovian planets should not be scarred by the large number of impact craters that dominate the faces of the Moon and Mars; however, there may be some contamination in the case of the satellites of Jupiter due to the diffusion of fragments from the asteroid belt. It is hoped that this anticipated scarcity of craters can be tested by future space probes.

\section{TEMPERATURE OF ACCRETION}

It is desirable to know whether the temperature that a given planetoid attained during the course of accretion was sufficient to melt it and thereby allow the differentiation of a core of dense material. The minimum temperature maintained by a planetoid in the act of accreting material is one whereby the energy inflow due to accretion is just balanced by the loss of blackbody radiation, or

$$
\frac{d E}{d t}=4 \pi R^{2} \sigma\left(T^{4}-T_{0}^{4}\right)=\frac{G M}{R}\left(4 \pi R^{2} \rho_{p}\right) \frac{d R}{d t}
$$


Using the accretion equation to evaluate $d R / d t$ and by minor algebra we find that

$$
T=\left\{\frac{\alpha G \rho_{p} \pi R^{2} V \rho_{a}\left[1+\left(R / R_{c}\right)^{2}\right]}{3 \sigma}+T_{0}\right\}^{4 / 4}
$$

Here $T_{0}$ is the blackbody temperature of the planetoid in the absence of accretion.

To evaluate $T$ we need to know $\rho_{a}$, the density of accretable material in the solar nebula. We have calculated this by assuming that the material presently in the terrestrial planets and asteroids, $M_{\text {total }}=2 M_{\oplus}$, was distributed uniformly in an annular sector of the solar nebula lying between 0.3 and $2 \mathrm{AU}$ from the Sun. The turbulent velocity $V$ determined the scale height of the dust above the plane of the nebula and consequently the density $p_{a}$ in the plane. For $V=0.02 \mathrm{~km} / \mathrm{s}$, we find that $\rho_{g}=1.6 \times 10^{-10} \mathrm{~g} / \mathrm{cm}^{3}$; whereas for $V=0.04 \mathrm{~km} / \mathrm{s}, \rho_{a}=0.8 \times 10^{-10} \mathrm{~g} / \mathrm{cm}^{3}$.

To illustrate the dependence of $T$ upon $R$, in figure 3 we have plotted this relation for a case in which $T_{0}=300 \mathrm{~K}$ and planetoid densities $\rho_{p}$ are $3.6 \mathrm{~g} / \mathrm{cm}^{3}$ and $5.5 \mathrm{~g} / \mathrm{cm}^{3}$. We note that if $T \geqslant 2 T_{0}$, then $T$ is practically independent of the particular $T_{0}$ chosen. We further note from the figure that Venus and Earth with $T \sim 3 \times 10^{3} \mathrm{~K}$ were probably the only terrestrial planets that thoroughly melted. Mercury and Mars with $T \sim 1100$ to $1800 \mathrm{~K}$ only partially melted. The Moon with $T \sim 600$ to $1000 \mathrm{~K}$ probably did not melt from accretion, and Ceres with $T \sim 303$ to $320 \mathrm{~K}$ was essentially accreted cold.

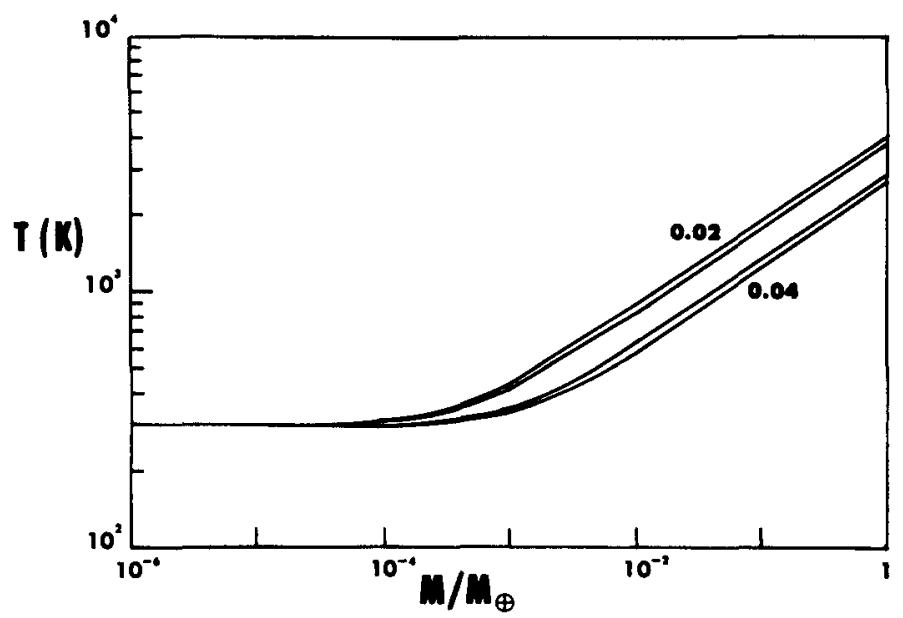

Figure 3.-Maximum temperatures attained by planetoids as the result of accretion. Curves are drawn for turbulent velocities of 0.02 and $0.04 \mathrm{~km} / \mathrm{s}$. The upper curve in each pair is for $\rho_{p}=5.5 \mathrm{~g} / \mathrm{cm}^{3}$ and the lower is for $\rho_{p}=3.6 \mathrm{~g} / \mathrm{cm}^{3}$. 


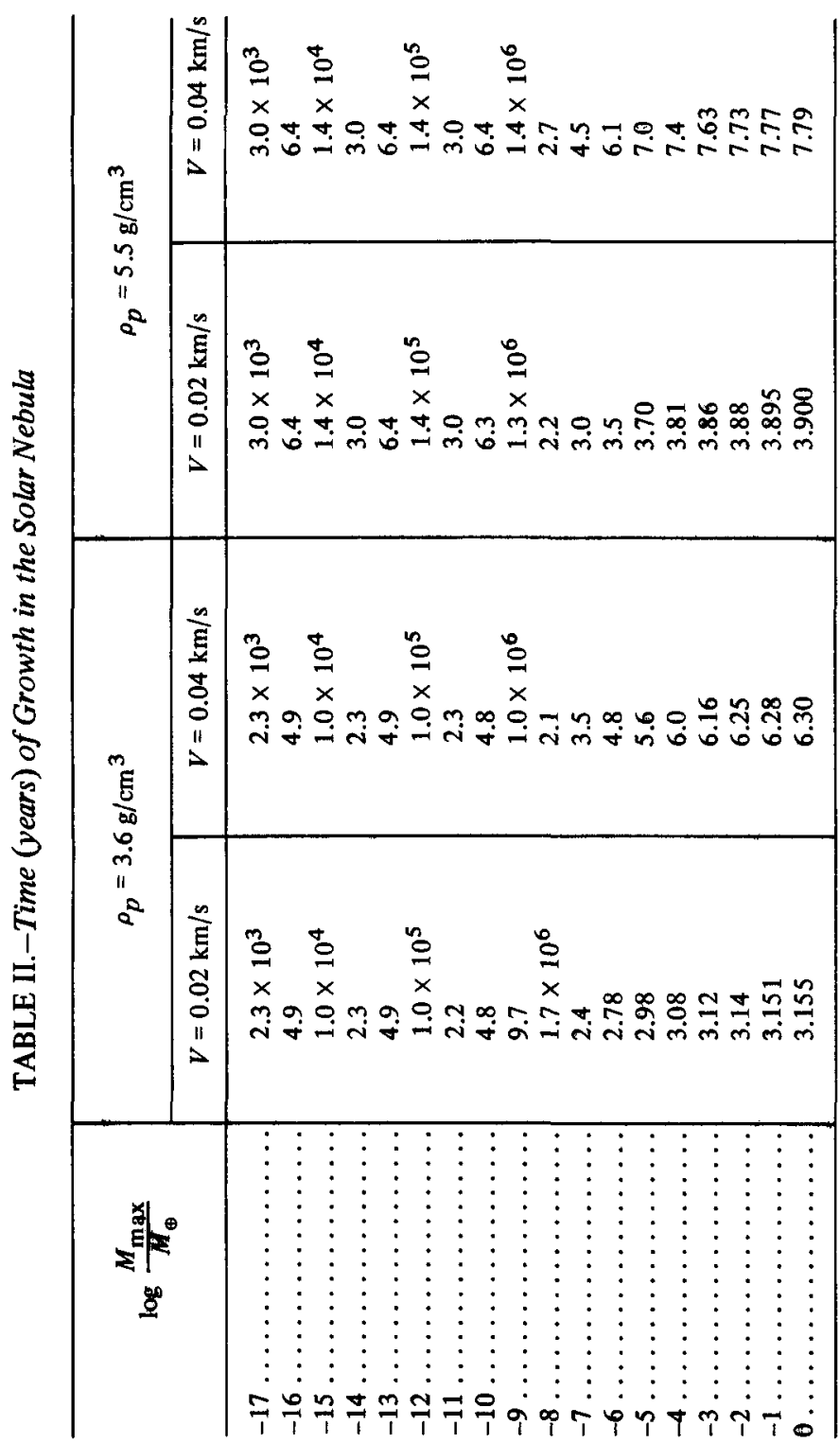


Thus an Earth-type core is expected on Venus but probably not on any of the other terrestrial planets. Because of their low accretion temperatures, the asteroids can be expected to have preserved the chemical integrity of the material that they accreted. Thus future onsite inspections of asteroid fragments may yield valuable insight into the chemical and physical properties of the preplanetoid material and, consequently, insight into the chemical and thermal properties of the solar nebula during the time of planetoid formation.

\section{TIME OF FORMATION}

If the seed bodies were formed at a uniform rate in time, as has been assumed, the average number of planetoids with radii in some range $R_{1}$ to $R_{2}$ remained constant even in the presence of further accretion as long as the radius $R_{\mathrm{max}}$ of the most massive planetoid in the system was greater than or equal to $R_{2}$. Thus at any given time during the accretion of the planetoids, their radius distribution function was the same as given in table I up to radius $R_{\max }$.

To find the time required for the radius of the largest planetoid in the system to grow to $R_{\max }$, we integrate equation (4). This gives

$$
t=\frac{\left(6 \rho_{p} / \pi G\right)^{1 / 2}}{\rho_{a} \alpha} \tan ^{-1} \frac{R_{\max }}{R_{c}}
$$

We note that, unlike the radius distribution function, this depends on the sticking coefficient $\alpha$ and the space density $\rho_{a}$ of accretable material. Setting $R_{\max }=\infty$, we see that formally a planet grows to infinite mass in a finite time

$$
t_{\infty}=\frac{\left(6 \rho_{p} / \pi G\right)^{1 / 2}}{\rho_{a} \alpha} \frac{\pi}{2}
$$

This is the characteristic time for forming a planetary system. We further note that a planetoid takes only twice as long to grow to $R=\infty$ as to grow to $R=R_{c}$.

Table II tabulates $t$ as a function of $R_{\max }$ for $\alpha=1$ and previously calculated $\rho_{a}$ values. The table shows that if $V=0.02 \mathrm{~km} / \mathrm{s}$, a planetoid only required about $8 \times 10^{4} \mathrm{yr}$ to increase its mass from that of Ceres to that of Earth. This suggests that if a stable seed body had formed about $8 \times 10^{4} \mathrm{yr}$ earlier in the asteroid belt, there might be a terrestrial planet there today. This small difference is less than 3 percent of the time required for a planetoid mass to grow to $1 M_{\oplus}$.

\section{REFERENCES}

Anders, E. 1965, Fragmentation History of Asteroids. Icarus 4, 399-408.

Hartmann, W. K. 1968, Growth of Asteroids and Planetesimals by Accretion. Astrophys. J. 152, 337-342. 
Hartmann, W. K., and Hartmann, A. C. 1968, Asteroid Collisions and Evolution of Asteroidal Mass Distribution and Meteoritic Flux. Icarus 8, 361-381.

Hills, J. G. 1970, The Formation of the Terrestrial Planets. Bull. Amer. Astron. Soc. 2, 320.

Piotrowski, S. 1953, The Collisions of Asteroids. Acta Astron. Ser. A 5, 115-138. 